\title{
Fixação externa do anel de llizarov para fraturas complexas do platô tibial
}

\section{Ilizarov Ring External Fixation for Complex Tibial Plateau Fractures}

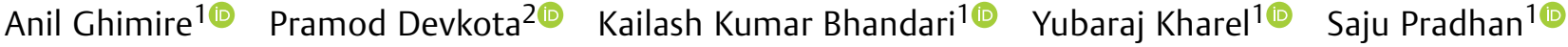 \\ ${ }^{1}$ Hospital Ortopédico do Nepal, Jorpati, Kathmandu 44601, Nepal and \\ Patan Academy of Health Sciences, Patan Hospital, Lalitpur 44700 \\ Nepal \\ 2 Departamento de Ortopedia e Cirurgia de Trauma, Patan Hospital, \\ Academia Patan de Ciências da Saúde, Lalitpur, Kathmandu, Nepal \\ Endereço para correspondência Pramod Devkota, MBBS MS (Ortho), \\ Department of Orthopaedics and Trauma Surgery, Patan Academy of \\ Health Sciences School of Medicine, Lalitpur, Nepal \\ (e-mail: devkotap@gmail.com).
}

Rev Bras Ortop 2022;57(4):667-674.

\section{Resumo}

Palavras-chave

- técnica de llizarov

- fixadores externos

- fraturas da tíbia

- classificação de Schatzker
Objetivo Analisar os resultados clínicos, radiológicos e funcionais da fratura do planalto tibial (Schatzker Tipo V, VI) tratada com fixador externo do anel Illizarov com ou sem abertura mínima.

Métodos Um total de 52 fraturas do planalto tibial dos tipos V e VI foram tratadas com fixador externo do anel Ilizarov com ou sem fixação interna mínima. A avaliação do desfecho funcional foi feita utilizando-se o escore da American Knee Society (AKS, na sigla em inglês) com consolidação clínica, radiológica e complicações encontradas. Resultados Foram 37 (71,15\%) pacientes do sexo masculino e 15 (28,84\%) do sexo feminino, com idade média de 39,07 $\pm 12,58$ anos. Acidentes de trânsito (ATs) foram a principal causa das fraturas, contabilizando 32 casos $(61,53 \%)$, seguidos por lesão por queda, com 16 casos (30,76\%), e impacto direto, com 4 casos (7,69\%). Foram 21 $(40,38 \%)$ casos de fraturas tipo V, 31 (59,61\%) casos do tipo VI e $24(46,15 \%)$ casos de fratura exposta. Os escores médios da AKS para as fraturas tipo V e VI foram de $82,8 \mathrm{e}$ 80,70 , respectivamente, mas não foi estatisticamente significativo em $p<0,05$. 0 escore médio da AKS para fraturas fechadas e abertas também não foi estatisticamente significativo em $p<0,05$.

Conclusões Para a fratura do planalto tibial complexa dos tipos V e VI de Schatzker, a fixação externa de llizarov é um método de tratamento seguro, econômico e eficiente que resulta em resultados satisfatórios.

Trabalho desenvolvido no Departamento de Ortopedia e Cirurgia de Trauma, Hospital Ortopédico do Nepal, Jorpati,Kathmandu, Nepal.

recebido

06 de Setembro de 2020

aceito

25 de Junho de 2021

Publicado on-line

Novembro 11, 2021
DOI https://doi.org/

10.1055/s-0041-1739171. ISSN 0102-3616. (c) 2021. Sociedade Brasileira de Ortopedia e Traumatologia. All rights reserved.

This is an open access article published by Thieme under the terms of the Creative Commons Attribution-NonDerivative-NonCommercial-License, permitting copying and reproduction so long as the original work is given appropriate credit. Contents may not be used for commercial purposes, or adapted, remixed, transformed or built upon. (https://creativecommons.org/ licenses/by-nc-nd/4.0/)

Thieme Revinter Publicações Ltda., Rua do Matoso 170, Rio de Janeiro, RJ, CEP 20270-135, Brazil 


\begin{abstract}
Keywords

- llizarov technique

- external fixator

- tibial fracture

- Schatzker classification

Objective To analyze the results of clinical, radiological, and functional outcomes of tibial plateau fracture (Schatzker Type V, VI) treated with Illizarov ring external fixator with or without minimum opening.

Methods A total of 52 tibial plateau fractures of type V, VI were treated with llizarov ring external fixator with or without mininum internal fixation were studied. Functional outcome assessment was done using the American Knee Society (AKS) score with clinical, radiological union, and complications were analyzed.

Results There were $37(71.15 \%)$ male and 15 (28.84\%) female patients, with a mean age of $39.07 \pm 12.58$ years old. Road traffic accidents (RTAs) were the major cause of fracture, accounting for 32 cases (61.53\%) followed by fall injury, with 16 cases (30.76\%), and direct impact, with 4 cases (7.69\%). Twenty-one (40.38\%) cases were type $V$ and 31 (59.61\%) cases were type VI fractures, and there were 24 (46.15\%) cases of open fracture. The mean AKS score of Type $V$ and Type VI fractures were 82.8 and 80.70 , respectively, but this was statistically not significant at $p<0.05$. The mean AKS score of closed and open fractures were also statistically not significant at $p<0.05$. Conclusions For Schatzker Types $\mathrm{V}$ and VI complex tibial plateau fractures, Ilizarov external fixation is a safe, cost-effective and efficient treatment method that presents a satisfactory outcome.
\end{abstract}

\section{Introdução}

As fraturas do planalto tibial são lesões complexas e uma das tarefas mais difíceis e controversas de manejo. ${ }^{1}$ Schatzker et al. $^{2}$ classificaram estas fraturas em seis tipos, e os tipos V e VI são geralmente causados por lesões de alta energia. Os problemas comumente encontrados nestas fraturas são cominuição condilar, depressão articular, envolvimento diafisário, lesão grave do tecido mole, dano neurovascular, lesões ligamentosas e síndrome compartimental aguda, e estão diretamente relacionados com a tomada de decisão na cirurgia e o prognóstico. ${ }^{3}$ Durante o manejo, a incapacidade de restaurar a congruência articular e a presença de lesões graves nos tecidos moles são os culpados pelos maus resultados. ${ }^{4} \mathrm{O}$ objetivo do manejo é a restauração da congruência e da estabilidade articular, a preservação da biologia óssea, o restabelecimento do eixo mecânico e a preservação do movimento. ${ }^{5} \mathrm{O}$ manejo conservador resulta em desfecho ruim e o método aberto tem complicações como necrose cutânea, alta chance de infecções de feridas, rigidez articular e, em algum momento, necessidade de múltiplos desbridamentos, artrodese e até amputação. ${ }^{6} \mathrm{O}$ desenvolvimento do sistema de estabilização menos invasivo (LISS) e de técnicas de galvanização minimamente invasivas também não resolveram esses problemas. ${ }^{7}$ Na literatura, não há consenso de um método específico para o manejo destas fraturas.

A técnica Ilizarov é um conceito de redução indireta da fratura com fixação biológica, com resultado satisfatório das fraturas tibiais proximais complexas, o que também foi o conceito de abertura da articulação do joelho. ${ }^{8}$ Nesta técnica, as fraturas são geralmente reduzidas de perto por tração ou método indireto e a redução é mantida por fixação externa ou interna antes da aplicação de um fixador externo transarticular. $^{9}$
A técnica Ilizarov do fixador externo do anel é uma das opções para lidar com as fraturas tibiais proximais complexas com graves lesões nos tecidos moles. ${ }^{10}$ Os méritos desta técnica são: redução fechada ou por miniacesso de fratura, o que diminui a chance de complicações de feridas e tecidos moles; carga funcional precoce e suporte de peso; fácil ajuste do alinhamento enquanto estiver no fixador e retorno precoce da função articular; e tecnicamente mais fácil do que outro fixador interno para a substituição de joelho, se isso for necessário para artrite pós-traumática no futuro. ${ }^{11} \mathrm{O}$ objetivo do presente estudo foi avaliar os desfechos radiológicos, clínicos e funcionais das fraturas do planalto tibial tipos V e VI de Schatzker, gerenciadas pela fixação externa do anel de Ilizarov.

\section{Materiais e Métodos}

O presente estudo retrospectivo foi realizado no Departamento de Ortopedia e Cirurgia de Trauma da nossa instituição e foi aprovado pelo Comitê de Revisão Institucional (número 103/076-77). De janeiro de 2013 a dezembro de 2017, foram analisadas 52 fraturas do planalto tibial dos tipos V e VI de Schatzker que foram gerenciadas com fixador Ilizarov com ou sem fixação interna mínima (mini-ORIF, na sigla em inglês). Os critérios de inclusão foram pacientes $>18$ anos $\mathrm{e}<65$ anos, fraturas do planalto tibial fechadas e abertas Schatzker tipo V e VI, operação realizada dentro dos 10 primeiros dias de lesão e tempo de seguimento de pelo menos 1 ano após a remoção do fixador Ilizarov. Os critérios de exclusão foram pacientes $<18$ anos e $>65$ anos, pacientes associados a lesões neurovasculares, politraumatismo, traumatismo craniano associado e pacientes submetidos a fasciotomia ou aplicação de outro fixador externo temporário anteriormente. Antes de realizar a cirurgia, gesso 


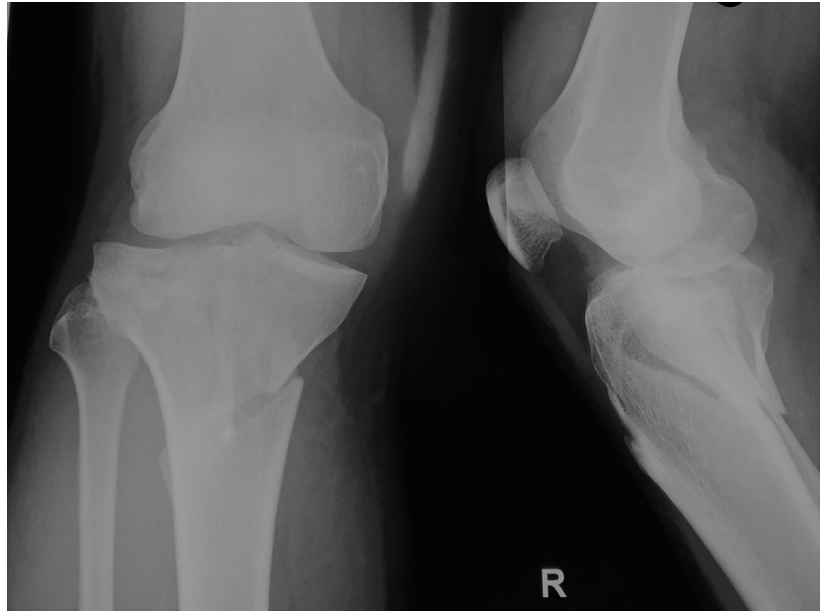

Fig. 1 Radiografias anteroposteriores (AP) e visão lateral da fratura tipo VI de um homem de 44 anos.

de Paris (POP, na sigla em inglês) foi aplicado até acima do joelho em todos os casos com a elevação adequada do membro. Se possível, foram feitas a irrigação primária e a cobertura das feridas com antibióticos intravenosos (IV) no pronto-socorro para todas as fraturas abertas. A cirurgia foi realizada no primeiro horário disponível de uma sala de cirurgia do departamento. Um dos cirurgiões seniores avaliou e decidiu aplicar o fixador do anel Ilizarov. Todos os dados foram coletados nos prontuários clínicos do hospital (-Fig. 1).

A demografia, união clínica e radiológica, mal alinhamentos e má uniões e complicações encontradas foram coletadas nos prontuários e analisadas. A função do joelho foi avaliada utilizando-se escore da American Knee Society (AKS, na sigla em inglês). ${ }^{12}$ Fraturas abertas foram classificadas de acordo com a classificação de Gustilo-Anderson. ${ }^{13}$

\section{Técnica Cirúrgica}

As cirurgias foram realizadas sob anestesia espinhal ou geral em uma mesa radiolúcida sob arco em C. Inicialmente, os fragmentos de fratura foram alinhados por meio de tração manual reta e a redução foi confirmada sob o arco em C. Fragmentos de fratura foram seguros com fórceps de patela ou fios $\mathrm{K}$ temporários. Um elevador de osso foi usado para elevar os fragmentos de fratura. Em alguns casos, os fragmentos articulares deprimidos precisavam de elevação usando mini-ORIF. A condição dos ligamentos e do menisco foi inspecionada, mas eles não foram tratados nesta fase. A redução do fragmento da fratura condilar foi feita e utilizaram-se fios olivados opostos entre si através dos fragmentos para alcançar a compressão interfragmentar - (Fig. 2). Às vezes, também se utilizaram fios olivados extras para a estabilização intra-articular. Os fios olivados foram utilizados para a compressão interfragmentar, aumentando a estabilidade do construto, permitindo distração gradual quando necessário e ajudando na redução de fragmentos em caso de tradução de fragmentos.

Três fios olivados foram utilizados de forma divergente de $60^{\circ}$ para estabilizar os fragmentos de fratura e fios olivados

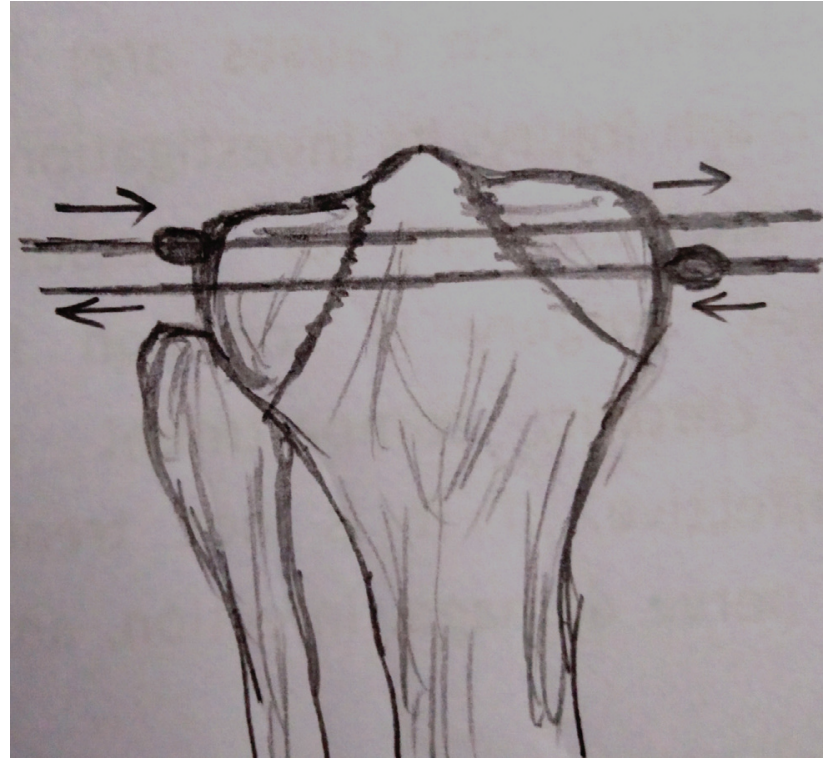

Fig. 2 Aplicação de fios olivados para a compressão interfragmentar.

de $1,8 \mathrm{~mm}$ foram inseridos da direção lateral para medial ligeiramente anterior à cabeça fibular para fornecer compressão interfragmentar. 0 primeiro anel foi fixado ao primeiro fio por dois parafusos de fixação, formando compressão rígida entre os fragmentos articulares; o outro fio foi aplicado da posição medial para a lateral ligeiramente distal do anel; ainda, um fio drop também foi inserido. ${ }^{10}$ Os anéis também foram ligados distalmente com outro anel por quatro hastes de interconexão. Tomou-se muito cuidado para restaurar o eixo mecânico em associação com os côndilos. A estrutura foi prolongada como um destracionador na extremidade distal do fêmur e foi fixada por dois meio-pinos entre o quadríceps e os isquiotibiais, e os anéis tibiais e femorais foram conectados por hastes de conexão. Isto foi feito apenas para casos selecionados quando o cirurgião sentiu a necessidade de manter a redução (-Fig. $\mathbf{3}$ ).

\section{Reabilitação pós-operatória}

A deambulação sem carga (DSC) precoce foi encorajada. 0 fisioterapeuta trabalhou a amplitude de movimento (ADM) ativa e ativa assistida e ensinou exercícios de fortalecimento muscular para o quadríceps e outros músculos. Indicou-se começar uma caminhada "toe tap" ou Frankenstein após uma semana. Foram feitas inspeções regulares dos curativos dos pinos e das feridas. A evolução de caminhada com peso parcial até a protegida com peso total aconteceu entre as 4aㅡ e a 6 semanas pós-operatórias. As marcações das consultas de acompanhamento foram feitas nas semanas 2,4 e 8 , nas marcas de 3, 6, 9, 12 e 16 meses e, posteriormente, conforme orientado pelo cirurgião até pelo menos 1 ano após a remoção do anel.

A consolidação das fraturas foi definida como a presença de ponte calus na radiografia sem movimento anormal no local da fratura, sem dor no uso de peso total e estresse no local da fratura após afrouxamento da estrutura. ${ }^{14}$ Se o paciente ainda tinha dor à deambulação com carga total, 


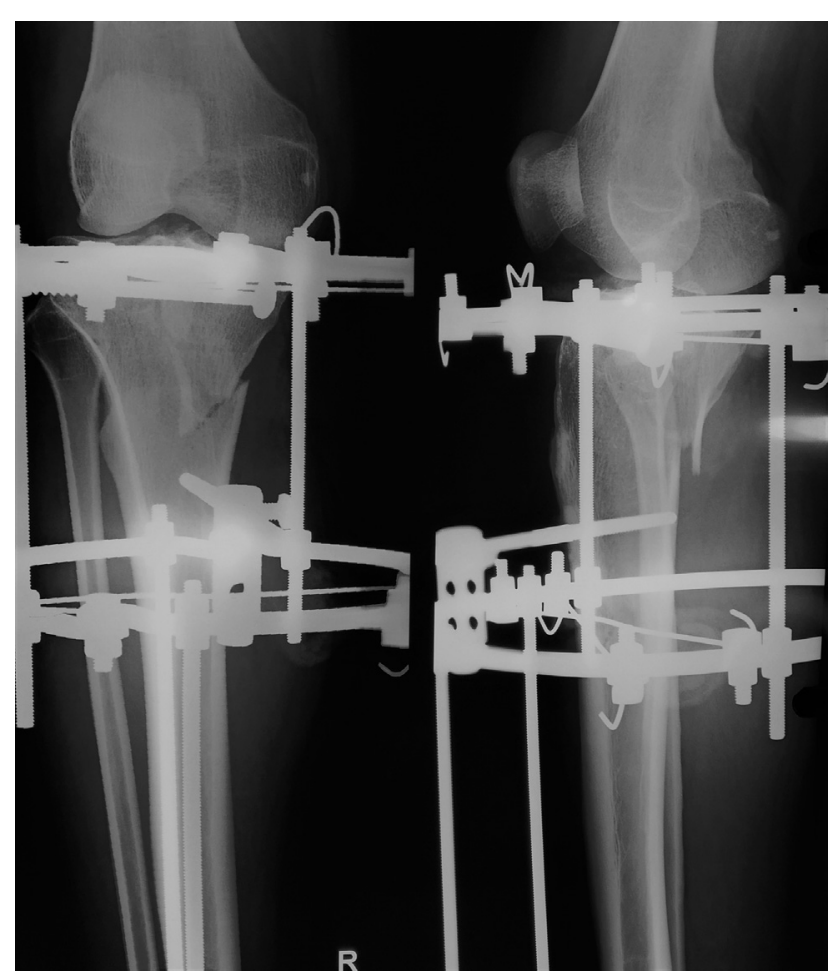

Fig. 3 Raio $X$ após fixação de llizarov com fixação interna mínima.

então o anel era apertado novamente e continuava-se com seguimento para sua remoção quando a deambulação com carga total fosse indolor.

\section{Análise estatística}

A análise estatística foi feita utilizando-se o IBM SPSS Statistics for Windows, versão 22.0 (IBM Corp., Armonk, NY, EUA). Dados quantitativos como idade, internação hospitalar, escore do joelho etc. foram expressos como média \pm desviopadrão (DP), enquanto dados qualitativos como gênero, causa de fraturas e fraturas abertas foram expressos em percentagem. $O$ teste $t$ de Student foi utilizado para análise de dados de dois grupos de variáveis contínuas com nível de significância em $p<0,05$ ( - Tabela 1 e - Fig. 4).

\section{Resultados}

O número total de casos de fraturas no planalto tibial que foram manejados cirurgicamente foi de 112, e 65 foram fraturas tipo V e VI. Sete pacientes foram manejados utilizando outras técnicas e 58 foram gerenciados por fixadores externos do anel Ilizarov com ou sem mini-ORIF. Seis casos foram excluídos por informações inadequadas dos prontuários. Assim, 52 pacientes permaneceram no estudo final. Todos os casos foram unilaterais e, entre eles, 37 (71,15\%) eram homens. O tempo médio de seguimento foi de $19,01 \pm 4,68$ meses (intervalo, 12 a 29 meses) após a remoção da estrutura. A média de idade dos pacientes foi de $39,98 \pm 13,02$ anos (faixa de 22 a 65 anos). Acidentes de trânsito (ATs) foram a principal causa das fraturas (61,53\%), seguidos por queda (30,76\%). Foram 24 (46,15\%) casos de fratura exposta, 21 (40,38\%) foram Schatzker tipo V, e 31 $(59,61 \%)$ foram fraturas de Schatzker tipo VI. Entre eles, 28 $(53,84 \%)$ foram fraturas fechadas e $24(46,15 \%)$ foram abertas.

Quarenta e dois casos foram tratados com 3 ou 2 anéis abaixo do joelho, 10 (19,23\%) casos tinham 1 anel acima do joelho, no fêmur distal, e 2 ou 3 anéis abaixo do joelho. Cinco $(9,61 \%)$ casos precisaram de mini-redução aberta e fixação interna com enxerto ósseo esponjoso, e a maioria dos casos foi acrescentada de parafusos esponjosos adicionais ou fios $\mathrm{k}$. O fixador do anel transjoelho foi removido em média 6,8 semanas após a cirurgia e iniciou-se a ADM do joelho.

O tempo médio de espera para a cirurgia após a lesão foi de 4,86 $\pm 2,11$ dias (intervalo, 1 a 10 dias) e o tempo médio de internação foi de $9,01 \pm 2,81$ dias (intervalo de 5 a 20 dias). 0 tempo médio para a consolidação e a remoção de todos os anéis externos foi de $15,09 \pm 2,25$ semanas (faixa de 12 a 20 semanas). A pontuação média da AKS foi de $81,65 \pm 8,52$ (faixa de 58 a 9), o grau médio de flexão do joelho foi de $110 \pm 13,22^{\circ}$ (faixa de 85 a $130^{\circ}$ ) e a extensão foi de $6,15 \pm 4,45^{\circ}$ (faixa de $0 \mathrm{a}-15^{\circ}$ ). 0 escore médio de AKS de fratura tipo $\mathrm{V}$ foi de $82,8 \pm 7,42$, e da fratura tipo VI foi de $80,70 \pm 9,10$. A fratura tipo $V$ apresentou melhor pontuação do que o tipo VI, mas não estatisticamente significativo $(p=0,2)$. 0 escore médio de fraturas fechadas da AKS foi de $80,07 \pm 9,49$, e as fraturas abertas foram de $83,33 \pm 6,82$, mas estatisticamente não significativas $(p=0,17)$. Detalhes do perfil dos pacientes são apresentados na -Tabela $\mathbf{1}$.

Infecções no trajeto dos pinos foram encontradas em 11 $(21,15 \%)$ casos que foram gerenciados com curativos regulares no trajeto dos pinos, educação da higiene pessoal para o paciente e um curto ciclo de antibióticos. No entanto, para gerenciar uma das infecções no trajeto do pino, o paciente foi internado no hospital e recebeu antibióticos intravenosos. Nenhuma artrite séptica e infecção profunda foram encontradas. Um mal alinhamento varo de $10^{\circ}$ foi encontrado em 5 casos. Um $(1,92 \%)$ paciente teve rigidez do joelho e $5(9,61 \%)$ pacientes tiveram atrofia do quadríceps, para a qual aconselhou-se fisioterapia vigorosa. Após a remoção do quadro, seis pacientes tiveram lesões do menisco ou dos ligamentos cruzados e foram gerenciados pela unidade artroscópica da nossa equipe. Também não foram encontrados casos de recorrência de fratura.

\section{Discussão}

Ilizarov introduziu o fixador externo do anel que evoluiu a nova compreensão da biologia óssea. ${ }^{15}$ Estas técnicas permitiram a correção de malformações ósseas rotacionais, angulares e translacionais, assim como a restauração da igualdade no comprimento dos membros. ${ }^{16}$ Os fixadores Ilizarov têm um sistema que consiste em muitas partes, permitindo sua aplicação a quase qualquer segmento de membro, tamanho e deformidade. ${ }^{17}$

As fraturas complexas do planalto tibial apresentaram alta incidência de complicações graves quando tratadas com técnica de fixação interna. ${ }^{2,18}$ A restauração da congruência articular é muito importante e o tratamento cuidadoso dos 
Fixação externa do anel de llizarov para fraturas complexas do platô tibial Ghimire et al. 671

Tabela 1 Detalhe do perfil do paciente com resultados clínicos

\begin{tabular}{|c|c|c|c|c|c|c|c|c|c|}
\hline \multirow{2}{*}{$\begin{array}{l}\text { Número } \\
\text { do caso }\end{array}$} & \multirow[t]{2}{*}{ Idade } & \multirow[t]{2}{*}{ Gênero } & \multirow{2}{*}{$\begin{array}{l}\text { Causa } \\
\text { da lesão }\end{array}$} & \multirow{2}{*}{$\begin{array}{l}\text { Classificação } \\
\text { Schatzker }\end{array}$} & \multirow{2}{*}{$\begin{array}{l}\text { Tipo } \\
\text { aberto }\end{array}$} & \multirow{2}{*}{$\begin{array}{l}\text { Tempo } \\
\text { fixador } \\
\text { em semanas }\end{array}$} & \multicolumn{2}{|c|}{ ADM do joelho } & \multirow{2}{*}{$\begin{array}{l}\text { Pontuação } \\
\text { da AKS }\end{array}$} \\
\hline & & & & & & & Flexão & $\begin{array}{l}\text { Defasagem } \\
\text { extensão }\end{array}$ & \\
\hline 1 & 41 & $M$ & AT & $\mathrm{VI}$ & Fechada & 12 & 100 & -5 & 85 \\
\hline 2 & 45 & $M$ & AT & $\mathrm{VI}$ & Fechada & 16 & 115 & 0 & 88 \\
\hline 3 & 32 & $\mathrm{~F}$ & AT & $\mathrm{V}$ & Fechada & 14 & 125 & -10 & 89 \\
\hline 4 & 60 & $\mathrm{~F}$ & Queda & $\mathrm{VI}$ & II & 17 & 130 & 0 & 87 \\
\hline 5 & 51 & $M$ & AT & $\mathrm{VI}$ & Fechada & 12 & 125 & -5 & 90 \\
\hline 6 & 40 & $\mathrm{M}$ & Queda & $\mathrm{VI}$ & Fechada & 14 & 125 & -5 & 86 \\
\hline 7 & 24 & $\mathrm{M}$ & Queda & $\mathrm{V}$ & III A & 14 & 120 & -5 & 92 \\
\hline 8 & 22 & $M$ & AT & $\mathrm{VI}$ & Fechada & 15 & 100 & -5 & 86 \\
\hline 9 & 32 & $\mathrm{M}$ & AT & $\mathrm{VI}$ & III A & 18 & 125 & -5 & 87 \\
\hline 10 & 61 & $\mathrm{M}$ & AT & $\mathrm{VI}$ & II & 13 & 100 & -5 & 88 \\
\hline 11 & 24 & $\mathrm{~F}$ & Queda & $\mathrm{VI}$ & III B & 20 & 100 & -10 & 89 \\
\hline 12 & 33 & $\mathrm{M}$ & AT & $\mathrm{V}$ & 1 & 14 & 120 & 0 & 94 \\
\hline 13 & 44 & $M$ & AT & $\mathrm{VI}$ & 1 & 14 & 120 & -5 & 84 \\
\hline 14 & 32 & $M$ & Queda & $\mathrm{V}$ & Fechada & 12 & 120 & 0 & 88 \\
\hline 15 & 32 & $\mathrm{M}$ & Queda & $\mathrm{VI}$ & Fechada & 12 & 125 & -5 & 87 \\
\hline 16 & 32 & $M$ & AT & $\mathrm{V}$ & III B & 14 & 100 & 0 & 85 \\
\hline 17 & 37 & $\mathrm{M}$ & Queda & $\mathrm{VI}$ & III A & 18 & 105 & -5 & 89 \\
\hline 18 & 26 & $M$ & Queda & $\mathrm{V}$ & Fechada & 16 & 90 & -5 & 86 \\
\hline 19 & 32 & $\mathrm{M}$ & Queda & $\mathrm{V}$ & Fechada & 14 & 120 & -10 & 87 \\
\hline 20 & 26 & $\mathrm{M}$ & AT & $\mathrm{VI}$ & 11 & 13 & 105 & 0 & 93 \\
\hline 21 & 50 & $M$ & Queda & $\mathrm{V}$ & Fechada & 13 & 125 & -10 & 91 \\
\hline 22 & 33 & $\mathrm{M}$ & AT & $\mathrm{VI}$ & II & 17 & 125 & -5 & 90 \\
\hline 23 & 45 & $\mathrm{M}$ & AT & $\mathrm{VI}$ & III A & 15 & 100 & -5 & 87 \\
\hline 24 & 65 & $\mathrm{~F}$ & Queda & $\mathrm{VI}$ & II & 14 & 120 & 0 & 89 \\
\hline 25 & 27 & $\mathrm{M}$ & Trauma direto & $\mathrm{V}$ & Fechada & 20 & 125 & -15 & 88 \\
\hline 26 & 32 & $\mathrm{~F}$ & Trauma direto & $\mathrm{V}$ & Fechada & 16 & 100 & -10 & 89 \\
\hline 27 & 60 & $\mathrm{~F}$ & Trauma direto & $\mathrm{V}$ & II & 18 & 115 & 0 & 85 \\
\hline 28 & 51 & $\mathrm{~F}$ & AT & $\mathrm{VI}$ & Fechada & 14 & 90 & -5 & 85 \\
\hline 29 & 40 & $\mathrm{~F}$ & AT & $\mathrm{V}$ & Fechada & 13 & 125 & -10 & 75 \\
\hline 30 & 24 & $\mathrm{~F}$ & Queda & $\mathrm{VI}$ & III B & 12 & 90 & -5 & 77 \\
\hline 31 & 22 & $\mathrm{~F}$ & AT & $\mathrm{V}$ & Fechada & 15 & 100 & -15 & 78 \\
\hline 32 & 32 & $\mathrm{~F}$ & AT & $\mathrm{V}$ & II & 16 & 120 & -5 & 79 \\
\hline 33 & 61 & $\mathrm{~F}$ & Queda & $\mathrm{VI}$ & Fechada & 17 & 110 & -10 & 80 \\
\hline 34 & 24 & $\mathrm{~F}$ & AT & $\mathrm{VI}$ & 1 & 19 & 125 & 0 & 81 \\
\hline 35 & 33 & $\mathrm{~F}$ & AT & $\mathrm{V}$ & Fechada & 12 & 90 & -15 & 82 \\
\hline 36 & 44 & $\mathrm{M}$ & AT & $\mathrm{VI}$ & Fechada & 14 & 120 & -5 & 83 \\
\hline 37 & 32 & $M$ & Queda & $\mathrm{V}$ & 1 & 13 & 110 & -5 & 82 \\
\hline 38 & 32 & $\mathrm{M}$ & AT & $\mathrm{V}$ & II & 16 & 115 & -5 & 77 \\
\hline 39 & 32 & $\mathrm{M}$ & AT & $\mathrm{V}$ & II & 14 & 90 & -10 & 76 \\
\hline 40 & 37 & $M$ & Queda & $\mathrm{VI}$ & III A & 17 & 90 & -10 & 75 \\
\hline 41 & 62 & $\mathrm{M}$ & AT & $\mathrm{VI}$ & Fechada & 19 & 100 & -5 & 78 \\
\hline 42 & 32 & $\mathrm{M}$ & AT & $\mathrm{VI}$ & 1 & 18 & 100 & -5 & 79 \\
\hline
\end{tabular}


672 Fixação externa do anel de Ilizarov para fraturas complexas do platô tibial Ghimire et al.

Tabela 1 (Continued)

\begin{tabular}{|c|c|c|c|c|c|c|c|c|c|}
\hline \multirow{2}{*}{$\begin{array}{l}\text { Número } \\
\text { do caso }\end{array}$} & \multirow[t]{2}{*}{ Idade } & \multirow[t]{2}{*}{ Gênero } & \multirow{2}{*}{$\begin{array}{l}\text { Causa } \\
\text { da lesão }\end{array}$} & \multirow{2}{*}{$\begin{array}{l}\text { Classificação } \\
\text { Schatzker }\end{array}$} & \multirow{2}{*}{$\begin{array}{l}\text { Tipo } \\
\text { aberto }\end{array}$} & \multirow{2}{*}{$\begin{array}{l}\text { Tempo } \\
\text { fixador } \\
\text { em semanas }\end{array}$} & \multicolumn{2}{|c|}{ ADM do joelho } & \multirow{2}{*}{$\begin{array}{l}\text { Pontuação } \\
\text { da AKS }\end{array}$} \\
\hline & & & & & & & Flexão & $\begin{array}{l}\text { Defasagem } \\
\text { extensão }\end{array}$ & \\
\hline 43 & 26 & M & Queda & V & Fechada & 14 & 115 & -5 & 83 \\
\hline 44 & 50 & $\mathrm{M}$ & AT & $\mathrm{VI}$ & Fechada & 13 & 125 & 0 & 60 \\
\hline 45 & 33 & $\mathrm{M}$ & AT & $\mathrm{VI}$ & Fechada & 15 & 100 & -10 & 65 \\
\hline 46 & 45 & $\mathrm{M}$ & AT & $\mathrm{VI}$ & 1 & 16 & 125 & -5 & 66 \\
\hline 47 & 65 & $\mathrm{M}$ & Trauma direto & $\mathrm{V}$ & Fechada & 18 & 100 & -10 & 67 \\
\hline 48 & 26 & $\mathrm{~F}$ & AT & $\mathrm{VI}$ & Fechada & 12 & 100 & -10 & 70 \\
\hline 49 & 50 & $M$ & AT & $\mathrm{VI}$ & Fechada & 15 & 125 & 0 & 72 \\
\hline 50 & 33 & $\mathrm{~F}$ & AT & $\mathrm{VI}$ & III A & 17 & 85 & -15 & 73 \\
\hline 51 & 45 & $M$ & AT & $\mathrm{V}$ & Fechada & 13 & 100 & -10 & 66 \\
\hline 52 & 65 & $M$ & AT & $\mathrm{VI}$ & Fechada & 18 & 90 & -15 & 58 \\
\hline
\end{tabular}

Abreviaturas: ADM, amplitude de movimento; AKS, American Knee Society; AT, acidente de trânsito.

tecidos moles também é importante. ${ }^{10}$ A maior vantagem do fixador externo Ilizarové sua capacidade de reduzir e segurar firmemente a fratura com ou sem dissecção mínima de tecido mole. ${ }^{3,11}$ Os fios tensionados Ilizarov lidam bem com o osso subcondral macio que atua como uma estrutura na reposição do osso esponjoso e evitam o colapso, reintegram a estabilidade essencial do local da fratura com uma manobra de ponte e permitem ao paciente transferir o peso através desta estrutura flexível para a diáfise distal, contornando a área cominuitada e permitindo o movimento e a sobreposição de peso na articulação enquanto é mantida a redução. ${ }^{3,19}$

No presente estudo, a média de idade dos pacientes foi de 39,07 anos, com predominância de pacientes do sexo mas-

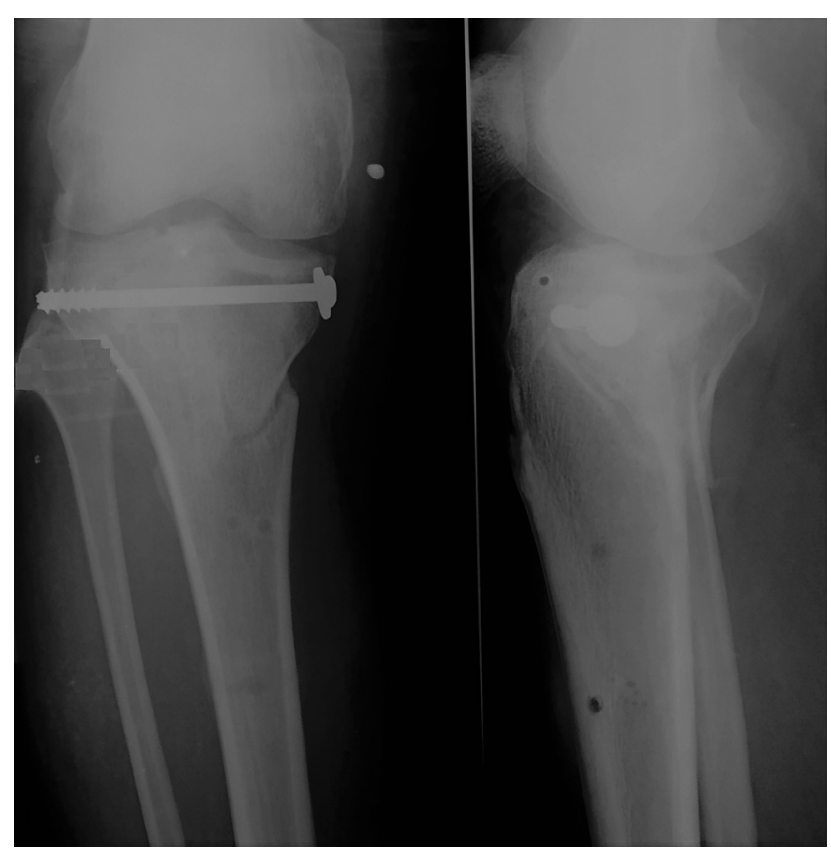

Fig. 4 Boa consolidação é vista após a remoção da estrutura Ilizarov com parafuso esponjoso canelado com arruela in situ. culino. Acidentes de trânsito foram a principal causa das fraturas, representando mais de $60 \%$. Ali relatou que a principal causa das fraturas foram ATs e houve predominância masculina, com uma idade média de 36 anos. ${ }^{20}$ Outros estudos também relataram que a principal causa da fratura foram ATs com predominância do sexo masculino, achados semelhantes aos nossos. ${ }^{1,3,11}$

O tempo médio de espera para a cirurgia após a lesão foi de 4,86 dias e o tempo médio de internação foi de 9,01 dias, com tempo médio de seguimento de 19,01 meses. Subramanyam et al. ${ }^{11}$ relataram que o tempo médio de espera para a cirurgia foi de 4 dias após a lesão, com um tempo médio de internação de 6 dias. Ali relatou um tempo médio de espera para a cirurgia de 3 dias pós-lesão, internação hospitalar de 8 dias e tempo de seguimento de 30 meses. No nosso estudo, o tempo de internação e operação foi ligeiramente maior, mas comparável aos destes estudos. No nosso estudo, 19,23\% dos casos apresentaram 1 anel acima do joelho e 5 casos precisaram de redução por miniacesso. 0 tempo médio para a remoção de todos os fixadores após a consolidação foi de 15,09 semanas. Ali ${ }^{20}$ relatou a aplicação de anel transjoelho para $30 \%$ dos casos e Subramanyam et al. ${ }^{11}$ aplicaram o anel transjoelho em 36,36\% dos casos, números ligeiramente superiores aos nossos, mas Catagni et al. ${ }^{21}$ relataram a aplicação de anel transjoelho em $67,79 \%$ dos casos. Subramanyam et al. ${ }^{11}$ realizaram o mini-ORIF em 7 casos do total de 30 de sua série, El Barbary et al. ${ }^{1}$ realizaram mini-ORIF em 6 de seus 30 casos, número semelhante aos do presente estudo. Mas Watson et al. ${ }^{22}$ relataram a necessidade de miniORIF para $79 \%$ dos casos, e Weiner et al. ${ }^{23}$ relataram $60 \%$ dos casos com necessidade de mini-ORIF. El Barbary et al. ${ }^{1}$ removeram a estrutura em uma média de 16,3 semanas e Catagni et al. $^{21}$ removeram a estrutura no tempo médio de 115 dias.

No presente estudo, a ADM média da flexão do joelho foi a flexão de $110^{\circ}$. Catagni et al. ${ }^{21}$ relataram flexão média do joelho de $119^{\circ}$ e Subramanyam et al. ${ }^{11}$ de $114^{\circ}$. Nossos resultados foram ligeiramente menores. 0 escore médio da 
AKS para fratura tipo $\mathrm{V}$ foi melhor do que o tipo VI, mas não estatisticamente significativo. Subramanyam et al. ${ }^{11}$ também relataram o escore estatisticamente não significativo da sociedade do joelho entre as fraturas dos tipos V e VI. O escore médio da AKS para fraturas abertas e fechadas também não foi estatisticamente significativo.

Infecções no trajeto dos pinos foram observadas em 11 $(21,15 \%)$ casos, os quais foram gerenciados com curativos regulares no trajeto dos pinos e um ciclo curto de antibióticos. Catagni et al. ${ }^{21}$ relataram $23,73 \%$ de infecção no trajeto do pino. Elsoe et al. ${ }^{24}$ relataram infecção no trajeto do pino em 33 dos seus 56 casos totais. Estes resultados foram semelhantes aos nossos. Não encontramos infecção profunda e osteomielite, e outros autores também relataram resultados semelhantes. ${ }^{11,21}$ Mal alinhamento varo de $10^{\circ}$ foi encontrado em 5 casos, resultado também semelhante aos demais achados. ${ }^{1,3,11,21}$

Foi relatada a aplicação de placa dupla para as fraturas complexas do planalto tibial com resultados satisfatórios em casos devidamente selecionados. ${ }^{25,26}$ Mas Bertrand et al. não relataram resultados estatisticamente diferentes entre ORIF e fixação externa híbrida em termos de consolidação, ADM do joelho e complicações. ${ }^{27}$ A Sociedade Canadense de Trauma Ortopédico também relatou resultados ligeiramente melhores de fixadores externos circulares sobre fixadores internos. ${ }^{28}$ Zhao et al. ${ }^{29}$ observaram vantagens dos fixadores externos sobre as fixações internas.

A placa pré-contornada do sistema LISS pode não ser adequada para todos os casos, e também aumenta o risco de dor e infecção associadas ao implante. ${ }^{21,30}$ Para países em desenvolvimento, estas placas também são muito caras, mas as estruturas Ilizarov são aplicáveis várias vezes, exceto os fios, o que também diminui a carga financeira para o paciente.

\section{Conclusão}

A técnica Ilizarov é uma das opções para o manejo de fraturas tibiais proximais complexas (tipos $\mathrm{VeVI}$ ) nas quais a redução aberta e a fixação interna não são adequadas devido à cominuição grave e lesões nos tecidos moles. É uma modalidade de tratamento segura, eficiente e amigável aos tecidos moles. Permite fixação estável, reabilitação precoce, suporte precoce de peso e cuidados com lesões de tecido mole com uma taxa de complicações relativamente menor.

\section{Contribuições dos Autores}

Ghimire A., Devkota P. e Bhandari K. K. contribuíram igualmente para a concepção e design da obra. Ghimire A. coletou os dados e Devkota P. e Bhandari K. K. participaram da análise e interpretação dos dados, da elaboração do manuscrito para conteúdo intelectual importante, e concordando em serem responsáveis por todos os aspectos do trabalho relacionados à sua exatidão e integridade. Kharel Y. e Pradhan S. contribuíram para a concepção da obra e a aquisição, análise e interpretação dos dados. Todos os autores leram e aprovaram o manuscrito final.

\section{Suporte Financeiro}

Sem apoio financeiro.

Conflito de Interesses

Os autores não têm conflitos de interesse para declarar.

\section{Agradecimentos}

Dr. Suman Kumar Shrestha, Dr. Nabees Man Singh Pradhan, Dr. Balakrishnan M Acharya da Academia Patan de Ciências da Saúde, Lalitpur, Nepal.

\section{Referências}

1 El Barbary H, Abdel Ghani H, Misbah H, Salem KEI Barbary HComplex tibial plateau fractures treated with Ilizarov external fixator with or without minimal internal fixation. Int Orthop 2005;29(03):182-185

2 Schatzker J, McBroom R, Bruce D. The tibial plateau fracture. The Toronto experience 1968-1975. Clin Orthop Relat Res 1979; (138):94-104

3 Debnath UK, Jha DK, Pujari PK. Results of ring (Ilizarov) fixator in high energy Schatzker type VI fractures of proximal tibia. J Clin Orthop Trauma 2018;9(02):186-191

4 Lansinger O, Bergman B, Körner L, Andersson GB. Tibial condylar fractures. A twenty-year follow-up. J Bone Joint Surg Am 1986;68 (01):13-19

5 John KS, Bret K. Wire ring fixation of complex tibial plateau fractures. In: Rozbruch SR, Ilizarov S, editors. Limb Lengthening and Reconstruction Surgery. New York: Information of Health Care; 2007:79-96

6 DeCoster TA, Nepola JV, el-Khoury GY. Cast brace treatment of proximal tibia fractures. A ten-year follow-up study. Clin Orthop Relat Res 1988;(231):196-204

7 Ozkaya U, Parmaksizoglu AS. Dual locked plating of unstable bicondylar tibial plateau fractures. Injury 2015;46(Suppl 2): S9-S13

8 Watson JT. High-energy fractures of the tibial plateau. Orthop Clin North Am 1994;25(04):723-752

9 Leunig M, Hertel R, Siebenrock KA, Ballmer FT, Mast JW, Ganz R. The evolution of indirect reduction techniques for the treatment of fractures. Clin Orthop Relat Res 2000;(375):7-14

10 Kumar A, Whittle AP. Treatment of complex (Schatzker Type VI) fractures of the tibial plateau with circular wire external fixation: retrospective case review. J Orthop Trauma 2000;14(05):339-344

11 Subramanyam KN, Tammanaiah M, Mundargi AV, Bhoskar RN, Reddy PS. Outcome of complex tibial plateau fractures with Ilizarov external fixation with or without minimal internal fixation. Chin J Traumatol 2019;22(03):166-171

12 Insall JN, Dorr LD, Scott RD, Scott WN. Rationale of the Knee Society clinical rating system. Clin Orthop Relat Res 1989;(248): 13-14

13 Gustilo RB, Anderson JT. Prevention of infection in the treatment of one thousand and twenty-five open fractures of long bones: retrospective and prospective analyses. J Bone Joint Surg Am 1976;58(04):453-458

14 Ranatunga IR, Thirumal M. Treament of Tibial Plateau Schatzker Type VI Fractures with the Ilizarov Technique Using Ring External Fixators Across the Knee: A Retrospective Review. Malays Orthop J 2010;4(02):34-39

15 Makhdoom A, Jokhio MF, Tahir SM, et al. Ligamentotaxis by Ilizarov Method in the Management of Tibial Plateau Fractures. World J Med Sci 2014;11(04):461-467

16 Ilizarov GA. Clinical application of the tension-stress effect for limb lengthening. Clin Orthop Relat Res 1990;(250):8-26

17 Velazquez RJ, Bell DF, Armstrong PF, Babyn P, Tibshirani R. Complications of use of the Ilizarov technique in the correction 
674 Fixação externa do anel de Ilizarov para fraturas complexas do platô tibial Ghimire et al.

of limb deformities in children. J Bone Joint Surg Am 1993;75(08): 1148-1156

18 Bennett WF, Browner B. Tibial plateau fractures: a study of associated soft tissue injuries. J Orthop Trauma 1994;8(03): 183-188

19 Ali AM, Burton M, Hashmi M, Saleh M. Outcome of complex fractures of the tibial plateau treated with a beam-loading ring fixation system. J Bone Joint Surg Br 2003;85(05):691-699

20 Ali AM. Outcomes of open bicondylar tibial plateau fractures treated with Ilizarov external fixator with or without minimal internal fixation. Eur J Orthop Surg Traumatol 2013;23(03):349-355

21 Catagni MA, Ottaviani G, Maggioni M. Treatment strategies for complex fractures of the tibial plateau with external circular fixation and limited internal fixation. J Trauma 2007;63(05): 1043-1053

22 Watson JT, Coufal C. Treatment of complex lateral plateau fractures using Ilizarov techniques. Clin Orthop Relat Res 1998;(353): 97-106

23 Weiner LS, Kelley M, Yang E, et al. The use of combination internal fixation and hybrid external fixation in severe proximal tibia fractures. J Orthop Trauma 1995;9(03):244-250

24 Elsoe R, Kold S, Larsen P, Petruskevicius J. A prospective observational study of 56 patients treated with ring fixator after a complex tibial fracture. Strateg Trauma Limb Reconstr 2017;12 (01):35-44
25 Khatri K, Lakhotia D, Sharma V, Kiran Kumar GN, Sharma G, Farooque K. Functional Evaluation in High Energy (Schatzker Type V and Type VI) Tibial Plateau Fractures Treated by Open Reduction and Internal Fixation. Int Sch Res Notices 2014; 2014:589538

26 Rohra N, Suri HS, Gangrade K. Functional and Radiological Outcome of Schatzker type V and VI Tibial Plateau Fracture Treatment with Dual Plates with Minimum 3 years follow-up: A Prospective Study. J Clin Diagn Res 2016;10(05):RC05-RC10

27 Bertrand ML, Pascual-López FJ, Guerado E. Severe tibial plateau fractures (Schatzker V-VI): open reduction and internal fixation versus hybrid external fixation. Injury 2017;48(6, Suppl 6) S81-S85

28 Canadian Orthopaedic Trauma Society. Open reduction and internal fixation compared with circular fixator application for bicondylar tibial plateau fractures. Results of a multicenter, prospective, randomized clinical trial. J Bone Joint Surg Am 2006;88(12):2613-2623

29 Zhao XW, Ma JX, Ma XL, et al. A meta-analysis of external fixation versus open reduction and internal fixation for complex tibial plateau fractures. Int J Surg 2017;39:65-73

30 Stannard JP, Wilson TC, Volgas DA, Alonso JE. The less invasive stabilization system in the treatment of complex fractures of the tibial plateau: short-term results. J Orthop Trauma 2004;18(08): 552-558 